\title{
Revisión sistemática de modelos de entrenamiento en rinoplastia
} Sistematic review of training models in rhinoplasty

Enrique A. LOZANO RUIZ*, Héctor O. MALAGÓN HIDALGO** Marcia R. PÉREZ DOSAL***

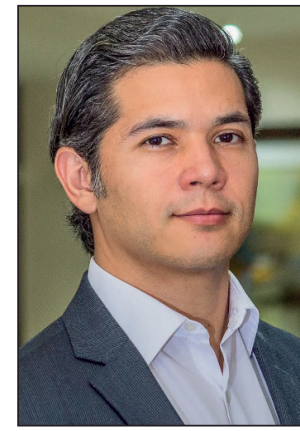

Lozano Ruiz E. A

\section{Resumen}

Antecedentes y Objetivo. La rinoplastia es uno de los procedimientos más frecuentes y más complejos de la Cirugía Plástica. Residentes de todo el mundo concuerdan en que su entrenamiento en rinoplastia es deficiente y en algunas ocasiones nulo. Algunos dicen no contar con la confianza suficiente para realizarla al finalizar su formación especializada. No todos los países establecen un método de entrenamiento estandarizado para la adquisición de experiencia y habilidades en rinoplastia, y si bien existen algunos modelos, no han sido evaluados y se desconoce su efectividad. Realizamos una revisión sistemática de las publicaciones que describen modelos de entrenamiento para aprendizaje, adquisición y/o mejora de habilidades en rinoplastia.

Material y método. Llevamos a cabo una revisión sistemática mediante búsqueda electrónica de la literatura en las diferentes bases de datos: MEDLINE Pubmed (1980 a junio de 2016), EMBASE Ovid (1946 a segunda semana de junio de 2016), LILACS Scielo (1982 a junio de 2016) usando como criterio de selección: estudios descriptivos sobre uso de modelos de entrenamiento en rinoplastia.

Resultados. Revisamos 6 modelos de entrenamiento publicados entre 2005-2014; 3 con tejido animal, 1 en cadáveres y 2 con materia sintético. Ninguno contaba con evaluación y validación de los modelos propuestos, por lo cual no es posible determinar si funcionan para mejorar las habilidades quirúrgicas durante la formación especializada en Cirugía Plástica.

Conclusiones. Aunque está demostrada la eficacia de los modelos de entrenamiento quirúrgico en otras especialidades y su uso es requisito para acreditación en EE. UU., no encontramos evidencia de la existencia de modelos eficaces en rinoplastia. Es por ello que nuestra revisión abre la puerta para la búsqueda de un modelo validado en nuestra especialidad.

Palabras clave Rinoplastia, Modelos educacionales, Entrenamiento quirúrgico simulado

\section{Nivel de evidencia científica Recibido [esta versión] Acentado}

\begin{abstract}
Background and Objective. Rhinoplasty is one of the most frequent and complex surgeries performed within the Plastic Surgery field. Residents around the world agree on a general deficiency from their rhinoplasty training and, in some cases, the absence of it. Someone, by the end of their training, still feel lack of confidence when performing a rhinoplasty. Currently, not all the countries have established a standardized training method for the acquisition of expertise and skills in rhinoplasty, and although there are several models, they have not been evaluated and therefore their efficiency as a training model is unknown.
\end{abstract}

Methods. We conduct a systematic review performing a systematic review of published articles about the theme. Search strategy included the following database: MEDLINE Pubmed (1980 to June 2016), EMBASE Ovid (1946 to second week of June 2016), LILACS Scielo (1982 to June 2016). Selection criteria used was: descriptive studies reporting the use of training models for rhinoplasty.

Results. Six training models, during a period from 2005-2014, were evaluated. Three were based on animal tissue, 2 on synthetic materials and 1 on a cadaveric model. No one had been evaluated or validated as to whether they improve surgical skills of residents in training.

Conclusions. Even do, in other medical specialties the effectiveness of surgical training models has been proven and such training is considered a requirement in the USA, within the rhinoplasty field no evidence of the effectiveness from these models was found. This revision delivers a path to continue with their effectiveness evaluation and skills improvement in Plastic Surgery.

$\begin{array}{lr}\text { Key words } & \begin{array}{l}\text { Rhinoplasty, Educational models, } \\ \text { Surgical training models }\end{array} \\ \text { Level of evidence } & \text { 4a Therapeutic } \\ \text { Received [this version] } & 29 \text { February2019 } \\ \text { Accepted } & \text { 24 July/2019 }\end{array}$

Conflicto de intereses: Los autores declaran no tener ningún interés financiero relacionado con el contenido de este artículo. Financiación: No hubo fuentes externas de financiación para este trabajo. 
Introducción

El modelo tradicional de aprendizaje quirúrgico se fundamenta en el principio de "aprender haciendo." A través de los años, el cirujano en entrenamiento encuentra una amplia gama de condiciones clínicas mediante las cuales va construyendo gradualmente las habilidades necesarias, fusionando conocimientos, juicio clínico y práctica de las diversas técnicas quirúrgicas. Dado que los pacientes reales están involucrados en cada punto de este proceso, las necesidades de aprendizaje de quien se encuentra en entrenamiento quedan inevitablemente subordinadas a las necesidades clínicas del paciente. El aprendizaje es por tanto un proceso oportunista, embebido en la práctica clínica. ${ }^{(1)}$ En sus inicios, el método de enseñanza de la rinoplastia popularizado en Nueva York por Fomon en 1942 consistía en conferencias a primera hora de la mañana seguidas de 2 cirugías en vivo y después disecciones en cadáver. Estos cursos tenían una duración de 6 semanas. Posteriormente Tardy, con una extraordinaria técnica y destreza comunicativa, elevó el nivel de la enseñanza de la rinoplastia utilizando diagramas fotográficos, disecciones en cadáver, así como demostraciones quirúrgicas de gran calidad. ${ }^{(2)}$

Diversas encuestas realizadas en EE.UU. en 2006 y 2009 reportan que el $70 \%$ de los residentes consideran la rinoplastia como uno de los procedimientos más complejos dentro de su formación especializada en Cirugía Plástica, motivo por lo cual es de su interés desarrollar una mayor experiencia en esta materia. ${ }^{(3,4)}$ El Comité de Revisión de Residencias del Consejo de Acreditación de la Educación Médica Superior (Residency Review Committee of the Accreditation Council for Graduate Medical Education) establece un mínimo de 6 rinoplastias realizadas durante la etapa de formación y entrenamiento de los residentes en EE.UU. Sin embargo, entre los residentes que han logrado este número se percibe inconformidad con el modelo y el $80 \%$ de los encuestados consideran además que el número ideal de rinoplastias para su entrenamiento debería ser de 10 o más para poder llevar a cabo una rinoplastia en paciente real de una forma segura y con confianza. ${ }^{(3)}$ Todo esto indica que es un buen momento para modificar este requerimiento que se ha mantenido como tal durante 2 décadas.

Diversos estudios llevados a cabo en Brasil, Italia, Alemania, Inglaterra y Canadá enfatizan sobre la importancia de lograr un entendimiento en el entrenamiento en Cirugía Estética para preparar a los cirujanos plásticos para la demanda y retos a los que se enfrentarán en el momento actual..$^{(5)}$ A diferencia de lo que sucede en EE.UU., en Canadá no existe un número definido de rinoplastias como requerimiento mínimo para la acreditación de los residentes, y menos del 20\% de los cirujanos graduados en este país se considera adecuadamente preparado para integrar la Cirugía Estética en su futura práctica, a la vez que consideran la rinoplastia y la ritidectomía como los procedimientos más desafiantes de su recién adquirida especialidad. ${ }^{(6)}$

Al igual que en Canadá, en Alemania no existe un requerimiento establecido del número mínimo de procedimientos para que los residentes puedan acreditarse. El porcentaje de los que no han realizado procedimientos estéticos durante su formación especializada es del $10.2 \%$ y un $33 \%$ ha llevado a cabo no más de 10 casos de Cirugía Estética. Para el caso de la rinoplastia, el 85\% de los residentes no se consideran capaces de realizar dicho procedimiento, mientras que un 54\% considera su entrenamiento en Cirugía Estética como deficiente. ${ }^{(7)}$

Según datos estadounidenses la tasa de cirugías de revisión tras una rinoplastia primaria se encuentra en torno al $5-12 \%{ }^{(8)}$ En un examen critico de sus resultados, Rohrich, ${ }^{(9)}$ en sus 25 años de experiencia en rinoplastia primaria, declara que las metas de esta intervención se alcanzan a medida que se adquiere más experiencia, y presenta una tasa de revisión de rinoplastia primaria extraída de su propia práctica del $85 \%$ durante los primeros 5 años, del $15 \%$ en los siguientes 10 años, del $5 \%$ en los últimos 10 años, hasta llegar al 3.5\% en el momento de la publicación de su revisión.

Debido a la alta tasa de rinoplastias de revisión y la considerable curva de aprendizaje de las técnicas, se requiere una enseñanza y una adquisición de destrezas adecuadas para el manejo de los tejidos, así como la utilización de técnicas de sutura especialmente diseñadas para resolver problemas específicos, preferentemente antes de que el residente en entrenamiento inicie su practica en pacientes.

La enseñanza de la rinoplastia en México es muy variada. Una encuesta verbal entre los residentes de las diferentes escuelas de Cirugía Plástica comprobó que ninguna de las 14 escuelas de Cirugía Plástica del país cuenta con prácticas de rinoplastia en cadáveres como parte de su esquema de entrenamiento, y a pesar de no recoger una medición exacta, se sabe que existe un número de residentes que no llegan a realizar ninguna rinoplastia como parte de su entrenamiento. En México, como en otros países, es preocupante la deficiencia en la oportunidad de adquisición de experiencia quirúrgica de los residentes, lo cual, desde nuestro punto de vista, se traduce en una área de oportunidad para la investigación y el desarrollo de nuevos modelos de entrenamiento práctico.

Los simuladores proveen ambientes de aprendizaje realistas y seguros, respaldados por la retroalimentación y las mediciones objetivas del desempeño. Ofrecen de esta forma la oportunidad de adquirir y evaluar habili- 
dades a través de la práctica repetitiva en un ambiente seguro. ${ }^{(10)}$ Las habilidades son el corazón de la práctica quirúrgica; requieren el desarrollo de competencias psicomotoras en un proceso basado en la práctica regular. Las investigaciones sobre la adquisición de la experiencia, la mayoría de ellas desarrolladas en el campo no médico (deportes y música), han establecido los siguientes principios clave: ${ }^{(11,12)}$

1. La experiencia se adquiere únicamente mediante la práctica deliberada a través de muchos años, complementada por la retroalimentación que aporta el experto.

2. La motivación es crucial. La repetición de la tarea debe estar respaldada por la determinación de obtener una mejoría.

3. Es esencial tener un programa constante de entrenamiento.

Algunas de las ventajas clave de utilizar la simulación son las siguientes:

- La agenda de entrenamiento puede determinarse en función de las necesidades del aprendiz, no de las del paciente. Los aprendices pueden enfocarse en todos los procedimientos o componentes específicos, practicándolos tan frecuentemente como sea necesario. ${ }^{(13)}$

- Dado que el ambiente es seguro, los aprendices tienen "permiso para fallar" y para aprender de dicha fallo de una forma que sería impensable en un escenario clínico. Esto da la oportunidad de explorar los límites de cada técnica en vez de tener que permanecer dentro de una zona clínica segura. ${ }^{(14)}$

- Los simuladores pueden proveer evidencia objetiva del desempeño, utilizando sus propias funcionalidades de trazabilidad para mapear con todo detalle la trayectoria del aprendiz.

La educación médica basada en simulación con práctica direccionada ha demostrado ser superior a la educación tradicional a la hora de lograr la adquisición de habilidades clínicas específicas y de mejorar los resultados obtenidos en los pacientes en otros campos. ${ }^{(15)} \mathrm{La}$ aceptación, e incluso los requerimientos de la educación basada en la simulación, se han expandido cada vez más. En la actualidad, la simulación y los laboratorios de adquisición de habilidades son requerimientos del Consejo de Acreditación para la Educación Médica Superior en EE.UU. (ACGME, siglas por su nombre en inglés) para la acreditación de los programas de cirugía general, así como también es necesaria la participación en cursos de simulación para mantener la certificación por el Consejo Americano de Anestesiología. ${ }^{(16,17)}$

Hasta donde hemos podido comprobar, actualmente no existe en la literatura mundial una revisión sistemática de los modelos de entrenamiento en rinoplastia. La ense- ñanza de la rinoplastia en México se basa en la práctica de las técnicas y en la adquisición de destrezas directamente sobre los pacientes. En vista de que el entrenamiento en rinoplastia es restringido para algunos residentes y el promedio de procedimientos para otros es insuficiente, creemos que el tener un modelo de entrenamiento en esta materia sería una herramienta eficaz, reduciría la curva de aprendizaje y permitiría que los residentes practicaran en un ambiente seguro y sin riegos para los pacientes. Para ello, hemos creído necesario identificar previamente los modelos existentes y dar a conocer su efectividad o, llegado el caso mejorarlos o propiciar la creación de nuevos modelos para su implementación en las residencias de Cirugía Plástica en México.

En el presente estudio nos proponemos realizar una revisión sistemática de los artículos publicados en la literatura que describen modelos de entrenamiento para el aprendizaje, adquisición y/o mejora de habilidades en rinoplastia y valorar si alguno de ellos tiene una eficacia demostrada para este fin.

\section{Material y método}

Desarrollamos un protocolo del estudio basado en el proceso PRISMA por sus siglas en ingles (Preferred Reporting Items for Systematic Reviews and Meta-Analyses), item de referencia para publicar revisiones sistemáticas y metaanálisis. ${ }^{(18,19)}$ Dos investigadores independientes realizaron la búsqueda electrónica de la literatura en las bases de datos para identificar los estudios elegibles publicados hasta el 25 de junio de 2016, usando las palabras clave (Mesh y Decs) y sus combinaciones. Los límites de la búsqueda fueron: literatura en idioma inglés y español, estudios realizados en animales, cadáveres humanos y en modelos sintéticos. La Tabla I muestra las bases de datos empleadas para nuestra búsqueda.

Los criterios de inclusión empleados para la búsqueda fueron: resultados de la búsqueda de la literatura con términos relevantes: modelos de entrenamiento potencialmente útiles en Cirugía Plástica incluyendo aquellos utilizados por otras especialidades, por ejemplo, Otorrinolaringología para el manejo del septum nasal.

Los artículos excluidos fueron: aquellos que describen modelos de entrenamiento endoscópico, modelos de entrenamiento en los que no se practican técnicas de rinoplastia, modelos de entrenamiento para abordaje neuroquirúrgico, modelos de entrenamiento para cirugía de senos paranasales, modelos para entrenamiento virtual, modelos para planeación quirúrgica, estudios anatómico-radiológicos de nariz en animales y estudios duplicados. 
Tabla I.- Bases de datos electrónicas empleadas, estrategia de búsqueda y resultados

\begin{tabular}{|c|c|c|}
\hline Base de Datos & Estrategia de Busqueda & Resultado \\
\hline $\begin{array}{l}\text { MEDLINE } \\
\text { (Pubmed) }\end{array}$ & $\begin{array}{l}\# 20 \text { Search (I(I((Irhino surgery) OR nasal surgery) OR } \\
\text { nasal surgery procedure) OR rhinoplasty)) AND ((external } \\
\text { nose) OR nasal cartilage)) AND (((Ianimal models) AND } \\
\text { educational model) AND animal model) AND experimental } \\
\text { animal model)) AND ((((surgical training) OR surgical } \\
\text { training simulation) OR surgical training simulations) OR } \\
\text { surgical simulator) } \\
\# 19 \text { Search (((surgical training) OR surgical training } \\
\text { simulation) OR surgical training simulations) OR surgical } \\
\text { simulator } \\
\# 18 \text { Search (((animal models) AND educational model) } \\
\text { AND animal model) AND experimental animal model } \\
\# 17 \text { Search (external nose) OR nasal cartilage } \\
\# 16 \text { Search (((rhino surgery) OR nasal surgery) OR nasal } \\
\text { surgery procedure) OR rhinoplasty } \\
\# 15 \text { Search surgical Simulator } \\
\# 13 \text { Search surgical training simulations } \\
\# 12 \text { Search surgical training simulation } \\
\# 11 \text { Search surgical training } \\
\# 10 \text { Search experimental animal model } \\
\# 9 \text { Search animal model } \\
\# 8 \text { Search animal models } \\
\# \text { Search eductional model } \\
\# 6 \text { Search nasal cartilage } \\
\# 5 \text { Search external nose } \\
\# 4 \text { Search rhinoplasty } \\
\# 3 \text { Search nasal surgery procedure } \\
\# 2 \text { Search nasal surgery } \\
\# 1 \text { Search rhino surgery }\end{array}$ & 3 \\
\hline $\begin{array}{|ll|}\text { LILACS } & \text { via } \\
\text { Scielo } & \\
\end{array}$ & $\begin{array}{l}\text { \#4(tw:((tw:(nasal surgery)) OR (tw:(rhinoplasty)) OR } \\
\text { (tw:(rhino surgery)) OR (tw:(nasal surgery procedure)) )) AND } \\
\text { (tw:((tw:(animal model)) OR (tw:(animal models)) OR } \\
\text { (tw:(educational model)) OR (tw:(experimental animal } \\
\text { model)))) AND (tw:((tw:(surgica training)) OR (tw:(surgical } \\
\text { training simulation)) OR (tw:(surgical simulator)) OR } \\
\text { (tw:(surgical training simulations)))) } \\
\# 3 \text { (tw:(surgica training)) OR (tw:(surgical training simulation)) OR } \\
\text { (tw:(surgical simulator)) OR (tw:(surgical training simulations)) } \\
\# 2 \text { (tw:(animal model)) OR (tw:(animal models)) OR } \\
\text { (tw:(educational model)) OR (tw:(experimental animal model)) } \\
\# 1 \text { (tw:(nasal surgery)) OR (tw:(rhinoplasty)) OR (tw:(rhino } \\
\text { surgery)) OR (tw:(nasal surgery procedure)) }\end{array}$ & 1 \\
\hline $\begin{array}{l}\text { EMBASE } \\
\text { (Ovid) }\end{array}$ & 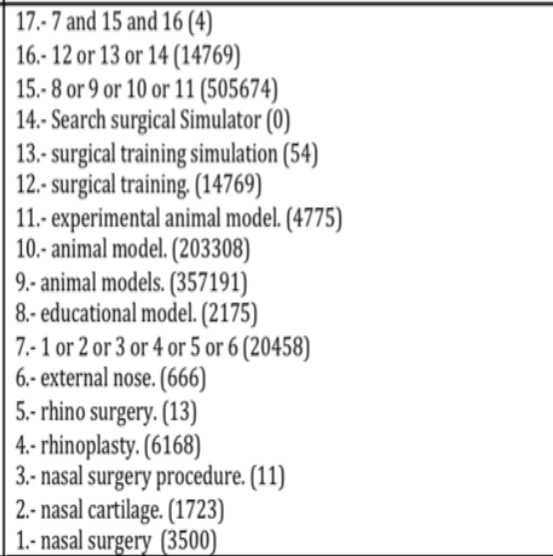 & 4 \\
\hline
\end{tabular}

Dos evaluadores participaron en la búsqueda de artículos de acuerdo a los criterios de inclusión y exclusión. Filtraron los títulos y resúmenes (abstracts) con las palabras clave y obtuvieron en su versión completa los estudios con datos insuficientes en título y resumen para analizar el contenido y tomar la decisión final de su inclusión o exclusión de nuestro trabajo (Tabla II).

\begin{tabular}{|c|c|c|c|c|}
\hline $\begin{array}{c}\text { Autores } \\
\text { Año de } \\
\text { publicación/ } \\
\text { envío }\end{array}$ & $\begin{array}{l}\text { Diseño del } \\
\text { estudio }\end{array}$ & $\begin{array}{l}\text { Características } \\
\text { población }\end{array}$ & N muestra & $\begin{array}{l}\text { Instrumento } \\
\text { medición }\end{array}$ \\
\hline $\begin{array}{l}\text { Patricio } \\
\text { Jacovella } \\
2005\end{array}$ & & Cadáver & 25 residentes & $\begin{array}{l}\text { Video grabación } \\
\text { prueba de } \\
\text { Strasser }\end{array}$ \\
\hline $\begin{array}{l}\text { Ghassan } \\
\text { Zabaneh } 2009\end{array}$ & Experimental & $\begin{array}{l}\text { Modelo sintético: } \\
\text { Silicón/ABS plástico. }\end{array}$ & No especifica & $\begin{array}{l}\text { Software } \\
\text { mimics y } \\
\text { autodesk } \\
\text { 3Dmax }\end{array}$ \\
\hline Bryce 2010 & Experimental & Modelo animal: pollos & $\begin{array}{l}4 \text { cartílagos } \\
\text { esternales de } \\
\text { pollo }\end{array}$ & Regla \\
\hline $\begin{array}{l}\text { David Chark } \\
2011\end{array}$ & Experimental & $\begin{array}{l}\text { Modelo animal: } \\
\text { Porcino }\end{array}$ & $\begin{array}{l}7 \text { cabezas de } \\
\text { cerdo }\end{array}$ & Caliper \\
\hline $\begin{array}{l}\text { Gal Moreira } \\
\text { Dini } 2012\end{array}$ & Experimental & $\begin{array}{l}\text { Modelo animal: } \\
\text { caprino }\end{array}$ & $\begin{array}{l}\text { No determina } \\
\text { con exactitud } \\
\text { el numero de la } \\
\text { muestra }\end{array}$ & Caliper \\
\hline $\begin{array}{l}\text { Alberto } \\
\text { Rancati } \\
2014\end{array}$ & & $\begin{array}{l}\text { Modelo animal: } \\
\text { caprino } \\
\text { Modelo } \\
\text { sintético(neoderma) }\end{array}$ & No determinado & Ninguno \\
\hline
\end{tabular}




\begin{tabular}{|c|c|c|c|c|c|c|}
\hline Intervención & $\begin{array}{c}\text { Variables independientes } \\
(\mathbf{x} 1 . . x n) / \text { variables } \\
\text { dependientes(outcome } \\
\text { y1..yn }\end{array}$ & $\begin{array}{l}\text { Variables } \\
\text { confusoras }\end{array}$ & $\begin{array}{c}\text { Pruebas } \\
\text { estadísticas } \\
\text { (multivariados) }\end{array}$ & Resultados (multivariado) & Conclusiones. & $\begin{array}{l}\text { Nivel de } \\
\text { gradiente } \\
\text { científico }\end{array}$ \\
\hline $\begin{array}{l}\text {-Disección anatómica para } \\
\text { familiarizarse con los tejidos. } \\
\text {-Familiarizar con la sensación manual } \\
\text { del abordaje nasal, resección del dorso } \\
\text { y osteotomías en cadáveres. } \\
\text { - Realizar rinoplastia primaria en } \\
\text { pacientes vivos. }\end{array}$ & $\begin{array}{l}\text {-Maniobras. } \\
\text {-Conocimiento. } \\
\text {-Posiciones. } \\
\text {-Habilidades especificas. }\end{array}$ & $\begin{array}{l}\text { No } \\
\text { determinadas }\end{array}$ & $\begin{array}{l}\mathrm{X} 2=28.68 \\
\mathrm{P}<0.001\end{array}$ & $\begin{array}{l}\text { En el grupo de estudio } 76 \% \\
\text { muy buenos, } 16 \% \text { bueno, } 8 \% \\
\text { aceptable. } \\
\text { En el grupo control } 4 \% \text { muy } \\
\text { bueno, } 32 \% \text { bueno, } 48 \% \\
\text { aceptable, } 16 \% \text { no aceptable }\end{array}$ & $\begin{array}{l}\text {-Permite identificar } \\
\text { estructuras anatómicas. } \\
\text {-Practicar la secuencia de } \\
\text { pasos de una rinoplastia. } \\
\text {-Permite la practica en } \\
\text { un ambiente controlado y } \\
\text { seguro. } \\
\text {-Permite errores. } \\
\text {-Provee el desarrollo de } \\
\text { sensación y habilidad } \\
\text { manual. } \\
\text {-Es un entrenamiento } \\
\text { eficiente y de bajo costo }\end{array}$ & $\mathrm{V}$ \\
\hline $\begin{array}{l}\text { Importancia de datos de tomografía } \\
\text { al software. } \\
\text {-Creación de modelos 3D virtual. } \\
\text { Creación de moldes con impresora } \\
\text { Zprinter } 450 .\end{array}$ & & $\begin{array}{l}\text { No } \\
\text { determinadas }\end{array}$ & No descrita & $\begin{array}{l}\text {-Creación de un modelo preciso } \\
\text { de rinoplastia } \\
\text {-La cubierta externa semeja } \\
\text { la piel. } \\
\text {-El silicón que simula los } \\
\text { cartílagos nasales se comporta } \\
\text { de manera similar a los humanos } \\
\text {-Se puede simular la mucosa que } \\
\text { cubre el septum }\end{array}$ & $\begin{array}{l}\text { El modelo creado tiene } \\
\text { el potencial de ser } \\
\text { utilizado como modulo } \\
\text { de entrenamiento para el } \\
\text { aprendizaje de rinoplastia }\end{array}$ & V \\
\hline $\begin{array}{l}\text {-Disección del cartílago esternal } \\
\text {-Tallado del cartílago. } \\
\text {-Unión de } 2 \text { cartílagos esternales con } \\
\text { cianocrilato para replicar el septum. } \\
\text {-Colocación dentro de fosa piriforme } \\
\text { en un cráneo humano. }\end{array}$ & $\begin{array}{l}\text { Peso/longitud máxima. } \\
\text {-Altura máxima }\end{array}$ & $\begin{array}{l}\text { No } \\
\text { determinadas }\end{array}$ & $\begin{array}{l}\text { Estadística } \\
\text { descriptiva } \\
\text { (promedios, } \\
\text { medias, modas, } \\
\text { mediana y } \\
\text { frecuencias). } \\
\text { IC, DS, ES } \\
\text { rangos. }\end{array}$ & $\begin{array}{l}\text {-Altura, longitud y grosor del } \\
\text { cartílago de pollo fue } 2.36 \mathrm{~cm} \text {, } \\
6.13 \mathrm{~cm} \text { y } 3.4 \mathrm{~mm} \text {. } \\
\text {-El humano fue de } 2.5 \mathrm{~cm}, 2.5 \\
\mathrm{~cm} \text { y } 3.25 \mathrm{~mm} . \\
\text {-De un cartílago se pueden } \\
\text { obtener } 2 \text { injertos spreader, } 1 \\
\text { injerto columelar, } 1 \text { injerto de } \\
\text { punta y } \\
\text { injertos de rim alar. }\end{array}$ & $\begin{array}{l}\text {-Permite el entrenamiento } \\
\text { simulado en un tejido } \\
\text { similar al humano. } \\
\text {-Provee la oportunidad de } \\
\text { practica para la formación } \\
\text { de injertos con formas } \\
\text { geométricas. } \\
\text {-Es fácil y económico. }\end{array}$ & $\mathrm{V}$ \\
\hline $\begin{array}{l}\text {-Toma de medidas de cruras en } \\
\text { cadáver. } \\
\text {-Creación de modelo virtual 3D con } \\
\text { ángulos y medidas obtenidos. } \\
\text { - Disección del tejido cartilaginoso, } \\
\text { corte de las cruras y formación del } \\
\text { modelo cartilaginoso mediante suturas } \\
\text { según modelo 3D. } \\
\text {-Fotografiado del modelo y medición } \\
\text { del Angulo de divergencia, distancia } \\
\text { interdomal y anchura domal derecha } \\
\text { e izquierda. } \\
\text {-Rehidratación del modelo con } \\
\text { solución salina con fosfato y } \\
\text { refrigerado a } 4 \text { grados centígrados. }\end{array}$ & $\begin{array}{l}\text { Dimensión anteroposterior } \\
\text { de crura media, intermedia } \\
\text { y lateral. } \\
\text {-Angulo de la orientación } \\
\text { cefálica. } \\
\text {-Grosor del cartílago. } \\
\text {-Distancia interdomal. } \\
\text {-Anchura domal. }\end{array}$ & & $\begin{array}{l}\text { Estadística } \\
\text { descriptiva }\end{array}$ & $\begin{array}{l}\text {-Dimensión anteroposterior } \\
\text { de crura media, intermedia y } \\
\text { lateral. } 4 \mathrm{~mm}, 6 \mathrm{~mm} \text { y } 10 \mathrm{~mm} \text {. } \\
\text { Angulo de la orientación } \\
\text { cefálica } 45 \text { grados . } \\
\text { Grosor del cartílago } 1 \mathrm{~mm}, \\
\text { distancia intermodal } 13.3 \mathrm{~mm} . \\
\text { Anchura domal } 6.2 \mathrm{~mm} .\end{array}$ & $\begin{array}{l}\text {-Fácil de construir y } \\
\text { es formado según las } \\
\text { especificaciones de } \\
\text { cadáveres humanos. } \\
\text {-La geometría de los } \\
\text { modelos formados semejan } \\
\text { una nariz tipo. Baxy tip } \\
\text { tipo III. } \\
\text {-Carece de cobertura } \\
\text { cutánea. }\end{array}$ & $\mathrm{V}$ \\
\hline $\begin{array}{l}\text {-Descongelar las cabezas por } 10 \text { min } \\
\text { en agua a } 68-77 \mathrm{~F} \text {. } \\
\text {-infiltración (hidrodiseccion) } \\
\text {-Incisión en V en columela. } \\
\text {-Disección de cartílagos nasales y } \\
\text { de dorso. } \\
\text {-Separación de los cartílagos alares. } \\
\text {-Incisión del ligamento interdomal. } \\
\text {-Separación de los cartílagos laterales } \\
\text { superiores del cartílago septal. } \\
\text {-Toma de injerto septal. } \\
\text {-Resección de giba cartilaginosa, } \\
\text { porción caudal del septum y cartílago } \\
\text { cuadrangular. } \\
\text {-Diseño y tallado de injertos. } \\
\text {-Colocación de injertos spreader. }\end{array}$ & $\begin{array}{l}\text {-Dimensión anteroposterior } \\
\text { de crura lateral. } \\
\text {-Longitud cefalocaudal. } \\
\text {-Grosor. } \\
\text {-Angulo de divergencia. } \\
\text {-Distancia interdomal- } \\
\text { Anchura domal. } \\
\text {-Grosor del proceso } \\
\text { ascendente maxilar. }\end{array}$ & $\begin{array}{l}\text { No se } \\
\text { identificaron }\end{array}$ & $\begin{array}{l}\text { Estadística } \\
\text { descriptiva }\end{array}$ & $\begin{array}{l}\text {-Dimensión anteroposterior de } \\
\text { crura lateral }(8 \mathrm{~mm}) \\
\text {-Longitud cefalocaudal }(12 \mathrm{~mm}) \\
\text {-Grosor } 1 \mathrm{~mm} \\
\text { Angulo de divergencia } 70 \\
\text { grados. } \\
\text {-Distancia interdomal } 13 \mathrm{~mm} . \\
\text { Anchura domal } 6 \mathrm{~mm} \\
\text {-Grosor del proceso ascendente } \\
\text { del maxilar }(0.9 \mathrm{~mm})\end{array}$ & $\begin{array}{l}\text {-Bajo costo(no especificado) } \\
\text {-Provee oportunidad de } \\
\text { entrenamiento. } \\
\text {-Tiene el potencial de } \\
\text { mejorar los resultados en las } \\
\text { rinoplastias. }\end{array}$ & $\mathrm{V}$ \\
\hline $\begin{array}{l}\text {-Marcaje. } \\
\text {-Infiltración. } \\
\text {-Disección ósea y cartilaginosa. } \\
\text {-Marcaje y resección de cartílagos } \\
\text { alares. } \\
\text {-Resección de giba ósea o } \\
\text { cartilaginosa. } \\
\text {-Osteotomías internas y externas. } \\
\text {-Preparación y colocación de injertos. } \\
\text {-Sutura de injertos y cierre de } \\
\text { incisión. }\end{array}$ & & & & & $\begin{array}{l}\text {-Permite el entrenamiento } \\
\text { de nuevas técnicas } \\
\text { quirúrgicas. } \\
\text {-El propósito es reducir la } \\
\text { curva de aprendizaje. } \\
\text {-Ganar experiencia en un } \\
\text { ambiente seguro. }\end{array}$ & V \\
\hline
\end{tabular}


Para cada estudio los datos obtenidos fueron: tipo de modelo, autor, marca comercial, aprendizaje, estructura formada, debilidades, reproducibilidad, evaluación del desempeño, costo (Tabla III).

Los dos evaluadores realizaron el análisis de cada uno de los artículos de forma independiente y finalmente el equipo de evaluación se reunió para resolver los puntos donde existieron dudas.
La búsqueda de la revisión electrónica fue de 8 artículos y mediante búsqueda manual obtuvimos 3 artículos; después de eliminar los duplicados obtuvimos 7 artículos de los cuales 1 fue excluido, resultando 6 artículos a texto completo para análisis (Gráfico 1), (Tablas II y III).

Analizando los modelos de entrenamiento existentes en la literatura encontramos lo siguiente:

Tabla III. Comparación de los modelos de entrenamiento en rinoplastia.

\begin{tabular}{|c|c|c|c|c|c|c|}
\hline Tipo & Cerdo & Cabra & Pollo & Cadáver & Sintético silicón & Sintético Neoderma \\
\hline Autor & David Chark & Gal Moreira Dini & $\begin{array}{l}\text { Adam Bryce } \\
\text { Weinfeld }\end{array}$ & $\begin{array}{l}\text { Patricio F. } \\
\text { Jacovella }\end{array}$ & Ghassan Zabaneh & Alberto Rancati \\
\hline Marca comercial & No aplica & No aplica & No aplica & No aplica & No aplica & $\begin{array}{l}\text { Rhinotrainer } \\
\text { (Prodelfus Co. } \\
\text { Brasil) }\end{array}$ \\
\hline Aprendizaje & $\begin{array}{l}\text { Sutura } \\
\text { interdomal }\end{array}$ & $\begin{array}{l}\text { Disección cartilaginosa } \\
\text { Disección septal } \\
\text { y toma de injerto } \\
\text { Resección de } \\
\text { giba cartilaginosa } \\
\text { Colocación de } \\
\text { spreaders } \\
\text { Resección cefálica } \\
\text { de alares Suturas } \\
\text { transdomales } \\
\text { e interdomales } \\
\text { Osteotomías }\end{array}$ & $\begin{array}{l}\text { Corte y tallado de } \\
\text { cartílago }\end{array}$ & $\begin{array}{l}\text { Abordaje } \\
\text { abiert, } \\
\text { Resección } \\
\text { de dorso } \\
\text { Osteotomías. }\end{array}$ & $\begin{array}{l}\text { Incisión } \\
\text { columelar } \\
\text { Disección de } \\
\text { colgajo cutáneo } \\
\text { Disección } \\
\text { estructuras } \\
\text { cartilaginosas } \\
\text { Resección } \\
\text { porción cefálica } \\
\text { de cruras laterales } \\
\text { Sutura interdomal } \\
\text { Disección y toma } \\
\text { de injerto septal } \\
\text { Cierre de abordaje }\end{array}$ & $\begin{array}{l}\text { Abordaje abierto o } \\
\text { cerrado } \\
\text { Disección de } \\
\text { cartílagos sintético } \\
\text { Medición y } \\
\text { resección de porción } \\
\text { cefálica de alares } \\
\text { Resección de giba } \\
\text { ósea y cartilaginosa } \\
\text { Osteotomías } \\
\text { internas y externas } \\
\text { Preparación y } \\
\text { colocación de } \\
\text { injerto } \\
\text { Postes en punta } \\
\text { nasal }\end{array}$ \\
\hline Estructura & $\begin{array}{l}\text { Formación } \\
\text { de cruras } \\
\text { nasales }\end{array}$ & $\begin{array}{l}\text { Spreaders } \\
\text { Columelar struts } \\
\text { Injertos de punta }\end{array}$ & $\begin{array}{l}\text { Formación de } \\
\text { septum nasal } \\
\text { Formación de } \\
\text { injertos struts y de } \\
\text { punta }\end{array}$ & $\begin{array}{l}\text { No se } \\
\text { especifica }\end{array}$ & Preformada & Preformada \\
\hline Debilidades & $\begin{array}{l}\text { Falta de } \\
\text { cobertura } \\
\text { cutánea }\end{array}$ & $\begin{array}{l}\text { Formación de } \\
\text { una estructura de } \\
\text { apariencia no similar a } \\
\text { la humana } \\
\text { Falta de cobertura } \\
\text { cutánea para } \\
\text { valoración de la } \\
\text { estructura formada }\end{array}$ & $\begin{array}{l}\text { Únicamente } \\
\text { permite el } \\
\text { desarrollo del } \\
\text { septum nasal }\end{array}$ & $\begin{array}{l}\text { Difícil } \\
\text { adquisición }\end{array}$ & $\begin{array}{l}\text { No se encuentra } \\
\text { disponible de } \\
\text { manera comercial }\end{array}$ & $\begin{array}{l}\text { Utilización de única } \\
\text { vez } \\
\text { Costo de simulador } \\
\text { elevado }\end{array}$ \\
\hline Reproducibilidad & $\begin{array}{l}\text { No } \\
\text { documentado }\end{array}$ & No documentado & No documentado & $\begin{array}{l}\text { No } \\
\text { documentado }\end{array}$ & No documentado & No documentado \\
\hline $\begin{array}{l}\text { Evaluación del } \\
\text { desempeño }\end{array}$ & Sin medición & Sin medición & Sin medición & Sin medición & Sin medición & Sin medición \\
\hline Costo & $\begin{array}{l}\text { No } \\
\text { especificado }\end{array}$ & No especificado & No especificado & No especificado & No especificado & $\begin{array}{l}\text { Estimado: } \$ 1,960.00 \\
\text { USD }\end{array}$ \\
\hline
\end{tabular}




\section{DIAGRAMA DE FLUJO DE RESULTADOS DE LA INCLUSIÓN DE ESTUDIOS}

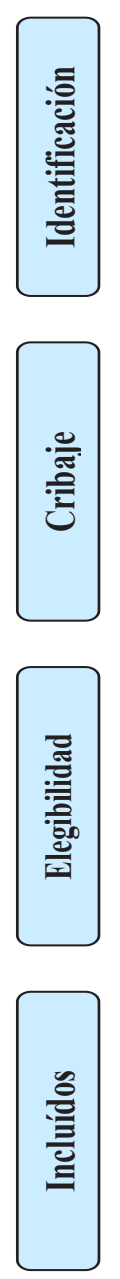

Resúmenes potencialmente relevantes identificados en la base de datos. $(n=8)$
Resúmenes potencialmente relevantes por otras fuentes. $(n=3)$
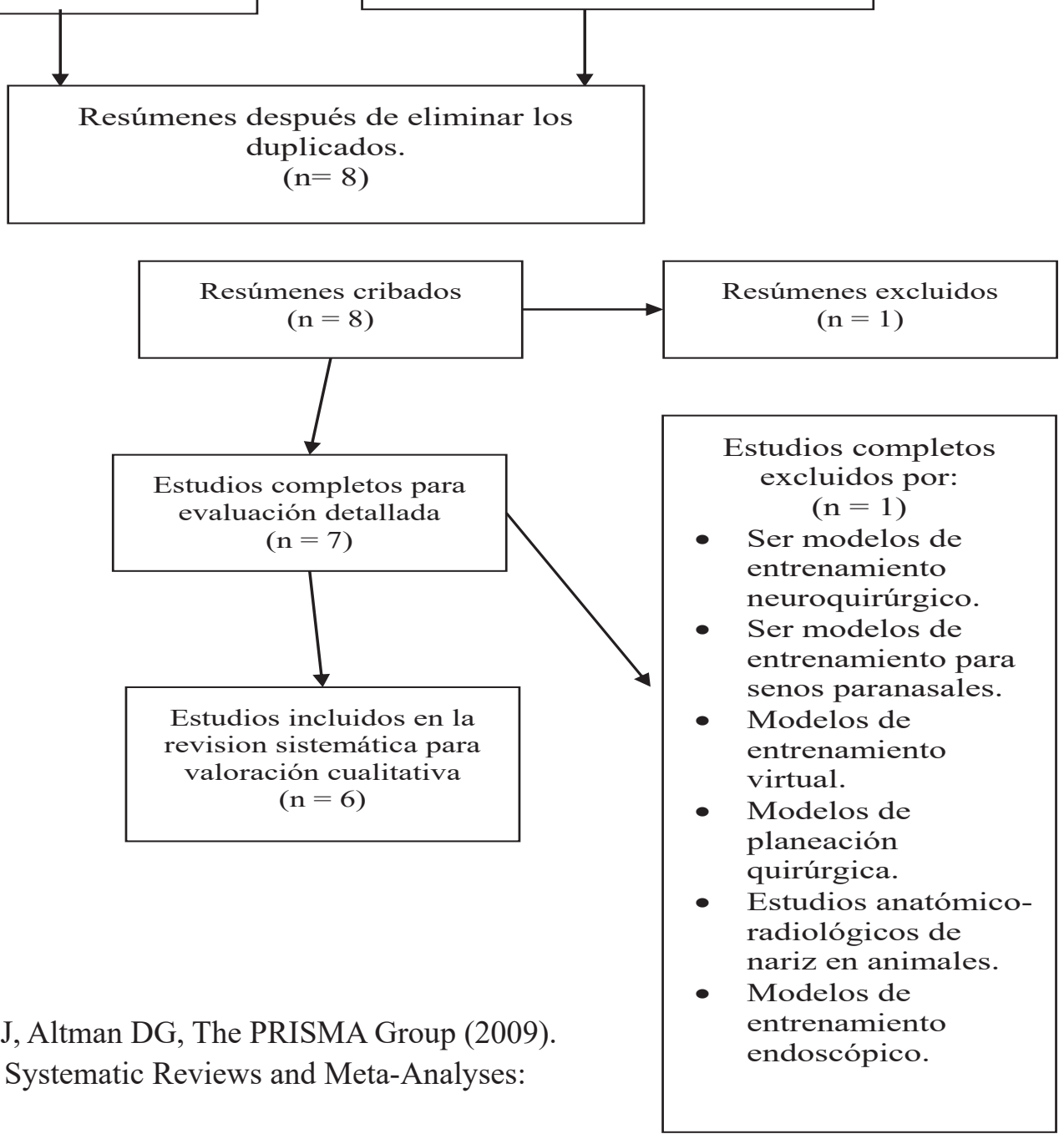

Moher D, Liberati A, Tetzlaff J, Altman DG, The PRISMA Group (2009). Preferred Reporting Items for Systematic Reviews and Meta-Analyses: The PRISMA Statement.

- De los 6 modelos de entrenamiento encontrados, 3 emplean tejido animal, 1 cadáveres y 2 material sintético.

- $\quad$ Los modelos fueron realizados entre 2005 y 2014.

- Ninguno ha llevado a cabo valoración de su efectividad ni ha sido validado.

\section{Discusión}

El entrenamiento en Cirugía Plástica Estética es limitado en México y sucede los mismo en otros países. Uno de los procedimientos quirúrgicos más desafiantes dentro de la especialidad es la rinoplastia y los residentes en formación necesitan poder completar su instruc- ción y adquirir las habilidades necesarias en sus técnicas, de gran complejidad, con la seguridad y capacidad de repetición que les pueden brindar modelos educacionales de entrenamiento apropiados. Las recomendaciones propuestas por algunos autores para mejorar el entrenamiento en rinoplastia claramente están en la línea de aumentar el número de procedimientos realizados por los residentes dentro de sus centros de formación, lo cual no es posible en todos los hospitales ni llevándolos a cabo sobre pacientes reales.

Sin embargo, nuestra revisión muestra la carencia de modelos de entrenamiento validados en rinoplastia y nos permite conocer la necesidad de realizar evaluaciones de los modelos existentes, así como la creación de otros 
nuevos que permitan adquirir las destrezas necesarias en un ambiente seguro para los pacientes y que a su vez sean accesibles para todos los resientes.

Patricio Jacovella ${ }^{(20)}$ desarrolló un programa de entrenamiento en cadáveres basado en 3 pasos: 1.- Familiarizar a los residentes con las estructuras nasales realizando disección anatómica de la nariz. 2.- Familiarizar al practicante con la sensación manual del abordaje nasal, de la resección cefálica y de la osteotomía lateral. 3.- Realizar posteriormente la cirugía nasal en pacientes, asistidos por profesores, para poner en práctica los conocimientos y habilidades obtenidos previamente. En su articulo no especifica los pasos de la técnica a seguir, los tipos de rinoplastia, las técnicas empleadas ni la reproducibilidad de las mismas. A nuestro juicio, una de las desventajas de este programa de entrenamiento es la falta de disponibilidad de cadáveres frescos en muchos servicios hospitalarios con residencia de formación médica especializada.

El modelo creado por el Ghassan Zabaneh ${ }^{(21)}$ realiza una importación de datos tomográficos de un paciente a un software llamado Mimics (Materialise, Leuven, Bélgica) y posteriormente lo edita con Autodesk 3D Max (Autodesk, San Rafael, California, EE.UU.), aislando las estructuras de la nariz para posteriormente formar los moldes de dichas estructuras mediante una impresora 3D. Una vez obtenidos los moldes se procede a la materialización de las estructuras a base de silicón simulando la piel, los cartílagos y la mucosa, y emplea plástico ABS para simular las estructuras óseas. Ya con el modelo formado, se pueden practicar los pasos de una rinoplastia, las incisiones columelares, la disección de las estructuras cartilaginosas, la sutura interdomal, la disección y la toma de injertos septales, así como la colocación de injertos en punta nasal y el cierre final de las incisiones.

El modelo de Adam Bryce Weinfeld ${ }^{(22)}$ utiliza cartílago de esternón de pollo para familiarizar al practicante con las características del cartílago septal; además permite el diseño y realización de injertos espaciadores, injertos alares, postes columelares e injertos de punta nasal. Es un modelo económico, aunque en su descripción no se especifica el costo, no se evalúa la reproducibilidad y no permite la formación de una estructura nasal completa.

El modelo propuesto por David Chark ${ }^{(23)}$ permite familiarizarse con las dimensiones y ángulos de las cruras nasales mediante un modelo generado por computadora que sirve como guía para posteriormente realizar las réplicas con tejido cartilaginoso de trompa de cerdo. En este modelo se realiza la unión de los cartílagos simulando las cruras medias con nylon 5-0. En los 7 especímenes que describe haber realizado se midieron los ángulos de divergencia, la distancia interdomal y la anchura domal.
Las ventajas de este modelo son su bajo costo (aunque el monto no se especifica en el artículo) y la adquisición de habilidad para la realización de suturas interdomales. En cuanto a sus desventajas, propone este modelo para la realización de técnicas de sutura para modificación de las cruras, pero encontramos que aunque las dimensiones y el grosor del cartílago son similares a las del humano, no lo es la maleabilidad y manipulación de este tipo de cartílago y no es posible la modificación de las cruras mediante técnicas de sutura; otra de sus desventajas es la falta de cobertura cutánea que permita visualizar el resultado final de la estructura cartilaginosa realizada.

Gal Moreira Dini(24) crea un modelo caprino para la simulación de los pasos en una rinoplastia: incisión columelar, disección del colgajo de piel del dorso y disección de los cartílagos nasales; evalúa también las dimensiones y los ángulos crurales, así como el grosor del proceso ascendente maxilar. Este modelo permite la disección y liberación de los cartílagos nasales, la disección y toma de injertos septales, la realización y colocación de injertos espaciadores (spreader grafts) y la resección cefálica de la crura lateral. Las suturas que se pueden practicar en este modelo son la transdomal y la interdomal. También es posible la colocación de injertos en punta, en escudo, subdomales y un poste columelar extendido de punta nasa. Una de sus grandes ventajas es que permite la práctica de osteotomías en el proceso ascendente maxilar. Sin embargo, este modelo no cuenta con cobertura cutánea similar a la del humano, y en su descripción no se mide la reproducibilidad de la técnica ni se especifica el costo del modelo.

Uno de los modelos más recientes para practica de rinoplastia es el Rinotrainer ${ }^{\circledR}$, un simulador de rinoplastia creado por la empresa Prodelphus en Brasil y publicado por Alberto Rancati. ${ }^{(25)}$ Está creado de un material llamado Neoderma que consta de 60 diferentes consistencias. Permite el entrenamiento de la infiltración, de los abordajes tanto abierto como cerrado, la disección de los tejidos, la resección de la giba ósea y de la cartilaginosa, las osteotomías internas o externas, la realización y colocación de injertos columelares, de injertos espaciadores (spreaders), injertos de listón alar (alar batten) y de injertos en dorso. En su artículo, el autor menciona que su objetivo es reducir la curva de aprendizaje a pesar de que aún no cuenta con estudios de evaluación de las destrezas adquiridas. Una de las desventajas de este modelo es su elevado coste, y aunque aún no se encuentra a la venta, un modelo similar, MAX politraumatizado (Prodelphus, Brasil) tiene un valor de 1.960 dólares, lo que lo hace inaccesible para la mayoría de los residentes en los Servicios de Cirugía Plástica ya que además solo puede usarse una vez. 
Como hemos analizado en nuestra revisión, el entrenamiento basado en simulación es una herramienta útil, y por lo tanto un área de oportunidad para resolver el importante déficit existente en la adquisición de habilidad quirúrgica en este campo tan desafiante de la Cirugía Plástica. El reto clave para el entrenamiento quirúrgico es proveer condiciones para un aprendizaje efectivo sin poner en riesgo la salud de los pacientes. Es por ello que, el entrenamiento a través de la simulación, es por sentido común atractivo; sin embargo, también es necesario saber si funciona.

Hasta donde pudimos valorar, ninguno de los modelos de entrenamiento para rinoplastia existentes en la literatura consultada cuenta con evaluación de las técnicas que promueven, por lo cual no es posible saber si dichos modelos funcionan para lograr la deseada mejoría en las habilidades quirúrgicas de los residentes en el caso de implementarse su uso de manera sistemática en las residencias de Cirugía Plástica.

Existen diversas herramientas para la evaluación de la adquisición de las destrezas quirúrgicas como el Objective Structured Assessment of Technical Skills (OSATS) ${ }^{(26)}$ en el cual se evalúan los siguientes puntos: 1) cuidado de los tejidos, 2) relación entre tiempo y movimientos, 3) manejo del instrumental, 4) conocimiento y familiaridad con los instrumentos, 5) flujo y progresión en el procedimiento, 6) uso y manejo de la ayuda del asistente quirúrgico y 7) conocimiento especifico de los pasos del procedimiento. Cada una de estas categorías consta de un rango del 1 al 5 para su evaluación, siendo el número 5 la calificación mayor para cada una.

Así mismo, los modelos de entrenamiento validados cuentan con comparación entre cirujanos expertos y los residentes de cada uno de los años de residencia para referencia de la eficiencia en el modelo. Una vez obtenida la puntuación y la comparación con los expertos se continua con evaluaciones posteriores para observar la mejoría de las destrezas alcanzadas. Cuando se ha visto que el modelo ha mejorado las habilidades de los residentes, el último paso sería comparar las habilidades de los residentes que han utilizado el modelo frente a las de los residentes que no han practicado en el modelo evaluado.

El aprendizaje basado en la simulación tiene un enorme potencial y pudiera revolucionar el entrenamiento basado en habilidades dentro de la práctica de la Cirugía Plástica, ayudando a reducir las curvas de aprendizaje, mejorando las técnicas de enseñanza, aumentando la confianza de los residentes en los diferentes procedimientos que aprenden y que luego van a realizar y salvaguardando la seguridad de los pacientes en el proceso de aprendizaje de los residentes en formación.
Conclusiones

Según nuestra búsqueda y comparación de modelos de entrenamiento en rinoplastia publicados en la literatura, los resultados de los estudios al respecto coinciden en la importancia del entrenamiento simulado para la obtención de los mejores resultados postquirúrgicos en los pacientes. Sin embargo, hasta donde hemos podido comprobar, aún no existe un simulador validado para el entrenamiento en rinoplastia, y no pudimos encontrar evidencia sobre cuál de los modelos de entrenamiento en rinoplastia es mejor o cuál es más efectivo realmente.

Sugerimos que es necesario llevar a cabo una investigación con una herramienta validada para la evaluación de las destrezas quirúrgicas adquiridas con dichos modelos, así como la creación de otros nuevos que permitan adquirir las destrezas necesarias en una técnica quirúrgica tan solicitada hoy en día en nuestra especialidad como es la rinoplastia, en un ambiente seguro para los pacientes y accesible para los especialistas en formación.

\section{Dirección del autor}

Dr. Enrique Aarón Lozano Ruiz

Centro Médico ISSEMYM Toluca

Avda. Baja Velocidad 284

San Jerónimo Chicuacalco, Metepec

Estado de México, México CP 52170

Correo electrónico: drenriquelozano@gmail.com

\section{Bibliografía}

1. Paisley AM, Baldwin PJ, Paterson-Brown S. Validity of surgical simulation for the assessment of operative skill. Br J Surg. 2001;88:1525-1232.

2. Simons R, Pham A, Greene R. Historia de la rinoplastia moderna, técnicas avanzadas en rinoplastia, en: Babak Azizzadeh, Edición 1, Barcelona, España, Elsevier, 2012. Pp. 1-19.

3. Morrison CM, Rotemberg SC, Moreira-Gonzalez A, Zins JE. A survey of cosmetic surgery training in plastic surgery programs in the United States. Plast Reconstr Surg. 2008;122:15701578

4. Georgette O. Cosmetic Surgery Training in Plastic Surgery Residency Programs in the United States: How Have We Progressed in the Last Three Years? Aesth Surg. J. 2011;31(4):445-455.

5. Momeni A, Kim RY, Wan DC, Izadpanah A, Lee GK. Aesthetic Surgery Training during Residency in the United States: A Comparison of the Integrated, Combined, and Independent Training Models. Plast Surg Int. 2014;2014:281-293.

6. Quinton J, Jamil A, Frank L, Warren R, Amr Y. Arkoubi, Raman C. et al. Cosmetic surgery training in Canadian plastic surgery residencies: are we training competent surgeons? Aesthet Surg J. 201; 33(1): 160-165.

7. Momeni A, Goerke SM, Bannasch H, Arkudas A, \& Stark GB. The quality of aesthetic surgery training in plastic surgery residency: a survey among residents in Germany. Ann of Plast Surg, 2013; 70(6), 704-708. 
8. Rohrich R. Rhinoplasty nasal surgery by the Masters, Ed. 3, Boca Raton, FL, CRC Press Taylor \& Francis Group, 2014. P. 775.

9. Rohrich R. Rhinoplasty nasal surgery by the Masters, Ed. 3, Boca Raton, FL, CRC Press Taylor \& Francis Group, 2014. Pp. 159-176.

10. Gorman PJ, Meier AH, Krummel TM. Simulation and virtual reality in surgical education. Real or unreal? Arch Surg 1999;134:1203-1208.

11. Ericsson KA. The acquisition of expert performance: an introduction to some of the issues. In: Ericsson KA, ed. The Road to Excellence: The Acquisition of Expert Performance in the Arts and Sciences, Sports and Games. Mahwah, New Jersey: Lawrence Erlbaum Associates, 1996, Pp.1- 50.

12. Ericsson KA, Krampe RT, Tesch-Romer C. The role of deliberate practice in the acquisition of expert performance. Psychol Rev 1993;100:363-406.

13. Shaharan S, Neary P. Evaluation of surgical training in the era of simulation. World J Gastrointest Endosc 2014; 6(9): 436-447.

14. Kneebone R. Simulation in surgical training: educational issues and practical implications. Medical Education 2003;37:267-277.

15. McGaghie WC, Issenberg SB, Cohen ER, Barsuk JH, Wayne DB. Does simulation-based medical education with deliberate practice yield better results than traditional clinical education? A meta-analytic comparative review of the evidence. Acad Med. 2011;86:706-711.

16. ACGME simulation requirement. www.acgme.org. Accessed March 29, 2011.

17. MOCA part IV: PPAI-simulation education courses. http:// www.theaba.org/pdf/Simulation_Education.pdf
18. The PRISMA statement for reporting systematic reviews and meta-analyses of studies that evaluate health care interventions: explanation and elaboration. http://www.annals.org/content/151/4/W-65.full. Accessed May 1, 2012.

19. Preferred reporting items for systematic reviews and meta-analyses: the PRISMA statement. http://www.annals.org/content/ 151/4/264.full. Accessed May 1,012.

20. Jacovella P, Developing Skills in Rhinoplasty Through Cadaver Training, Aesth Surg J 2005;25:643-645.

21. Zabaneh G, Lederer R, Grosvenor A, Wilkes G. Rhinoplasty: a hands-on training module. Plast Reconstr Surg 2009;124(3):952-954.

22. Weinfeld A, Chicken Sternal Cartilage for Simulated Septal Cartilage Graft Carving: A Rhinoplasty Educational Model, Aesth Surg J.2013. 30: 810.

23. Chark D, Oliaei S, Cyrus M, Wong B, Porcine Cartilage Model for Simulation of Nasal Tip Aesthetics and Mechanics, Aesth Surg J. 2011 31: 501.

24. Dini GM et al. A new animal model for training in rhinoplasty, Rev Bras Cir Plást. 2012;27(2):201-205.

25. Rancati et al. Rhinotrainer, Surgical Nose Simulator, Surgery Curr Res 2014, 4:6.

26. Martin JA, Regehr G, Reznick R. Objective structured assessment of technical skill (OSATS) for surgical residents. Br J Surg. 1997;84(2):273-278.

27. Moher D, Liberati A, Tetzlaff J, Altman DG, The PRISMA Group. Preferred Reporting Items for Systematic Reviews and Meta-Analyses: The PRISMA Statement. PLoS Med 2009; 6(7): e1000097. doi:10.1371/journal.pmed1000097. 